\title{
Structured Model Reduction of Power Systems
}

\author{
Christopher Sturk, Luigi Vanfretti, Federico Milano, and Henrik Sandberg
}

\begin{abstract}
This paper shows how structured model order reduction can be applied to power systems. For power systems divided into a study area and an external area, the proposed algorithm can be used to reduce the external area to a low order linear system, while retaining the nonlinear description of the study system. The reduction of the external area is done in such a way that the study system is affected as little as possible. It is shown that a lower model order can be attained when information about the study system is taken into consideration, than if the external system is reduced independently of it.

Index Terms-Model reduction of power systems, internal systems, structured model reduction
\end{abstract}

\section{INTRODUCTION}

Power systems today are colossal networks of interconnected power apparatus often spanning large geographical areas. With the growing trend in facilitating additional interconnections to neighbouring systems, the size and complexity of these networks will likely continue to increase, and thus, bring challenges for planning, operations and control of these networks. Hence, reduced-order models of power systems are desirable for many applications and studies, particularly for fast and cost-efficient stability analysis. Research on power system model reduction is extensive, and some methodologies focusing on specific applications have been implemented in software for automated model reduction. Generally speaking, model reduction of large power networks, typically known as Power System Dynamic Equivalencing [1], has the main aim of providing a system equivalent model able to reproduce the aggregated steady-state [2] and dynamic characteristics of the full-order network [3], while at the same time being compatible with the available computation tools for power system analysis [4], [5].

Model reduction techniques are often developed for specific applications such as: steady-state power flow for security assessment [6], small-signal stability analysis [7], transient stability [8], [9], network equivalents for electromagnetic transient studies [10], and control design applications [11]. In these applications the power system is divided into an internal

C. Sturk and H. Sandberg are with the Department of Automatic Control, School of Electrical Engineering, KTH, Royal Institute of Technology, SE10044 Stockholm, Sweden. E-mails: \{chsturk, hsan\} @kth.se. C. Sturk and H. Sandberg are supported in part by the Swedish Research Council under Grant 2007-6350 and Grant 2009-4565, and the Swedish Foundation for Strategic Research.

L. Vanfretti is with the Electric Power Systems Division, School of Electrical Engineering, Royal Institute of Technology (KTH), Teknikringen 33, SE-100 44, Stockholm, Sweden. E-mail: luigiv@kth.se. L. Vanfretti is supported by the STandUP for Energy collaboration initiative and the KTH School of Electrical Engineering.

F. Milano is with the Department of Electrical Engineering, University of Castilla - La Mancha, 13071 - Ciudad Real, Spain. E-mail: Federico.Milano@uclm.es. (or study system) and an external system. The internal or study system is usually set by the boundaries of the part of the system that a specific utility owns or it may be the area in which a particular power market is defined, usually this portion of the network is electrically close. However, it would be advantageous to arbitrarily set the area corresponding to the study system without any restrictions, which could aid in the design of power plant controllers and coordination of system protections. The external system is usually electrically distant, and in practice it is used to denote the service area of other utilities. The internal system is usually represented in detail while a reduced model is used for the external system.

From these approaches, there is particular interest in those that can preserve network structures and can be used for small signal analysis, transient stability analysis, and control design. Coherency based methodologies [1], [9], [12], [13] have been well accepted by the power engineering community and automated software for their application exist [4]. These methods start by identifying coherency in the generators of the power system [14], and in a second stage to proceed with the dynamic reduction of the system. The dynamic reduction process itself is carried out by aggregating the network [15] and aggregating the generators [16], [13]. At a later stage, it may even be possible to aggregate excitation controllers [17]. Although coherency methods have been accepted as the most reliable for power system dynamic equivalencing, the major drawback is that it may not always be possible to reduce specific parts of the power network, the nature of coherency is to cluster generator groups which imposes the areas in which the network can be divided. To overcome this limitation, some approaches that are capable of retaining a part of the network have been proposed [18], [19]

With the recent developments in power systems an increased interest has been seen from the automatic control community. Among other things it has been shown how model reduction algorithms popular in control can be applicable. These algorithms typically have a strong theoretical foundation and they are also very general in the sense that they are not targeted to a particular application. This makes them a good candidate for the reduction of power systems composed not only of synchronous machines but also of for instance renewable energy sources.

In [20] it is shown how Krylov subspace model reduction can be applied to the external area before reconnection with the study area. Principal component analysis (PCA) is another popular model reduction algorithm. In particular it is wellsuited for reduction of nonlinear systems. In [21] it was used to project the states of the external system onto a subspace of lower dimension. 
What we propose in this paper is to apply structured model order reduction to power systems with an external and a study area. The idea is to reduce the external system while trying to minimize the effect it will have on the study area. This is the main objective of structured model reduction, namely to reduce models locally while ensuring a small global model error.

Model reduction where various structural constraints are taken into account ("structured model reduction") has been considered in several papers. For example, in [22] frequencyweighted model reduction problems are considered, and in [23] controller reduction is considered. More general interconnection structures have been considered in, for example, [24], [25], [26].

The structure of the paper is as follows: In Section II we formulate the problem we want to solve. Section III summarizes the theory of structured model order reduction and in Section IV it is shown how this theory can be applied to power systems. Section V introduces the WSCC 3-machine, 9-bus system to which the proposed model reduction algorithm is applied.

\section{Problem Formulation}

We will assume that the power system we want to reduce can be divided into one part with system variables of interest to us, called the study area, and another part called the external area, that is only of interest in terms of its effect on the system variables in the study area (Fig. 1). This is motivated by the fact that one often only focuses on parts of the power system. Under this assumption the aim is to reduce the external area to a linear time-invariant (LTI) system of lower order, while retaining the nonlinear description of the study area. This is relevant since it allows for a physical interpretation of the reduced power system, which is helpful if one wants to simulate changes to the study area in terms of for instance transmission line failures or for the purpose of nonlinear control design.

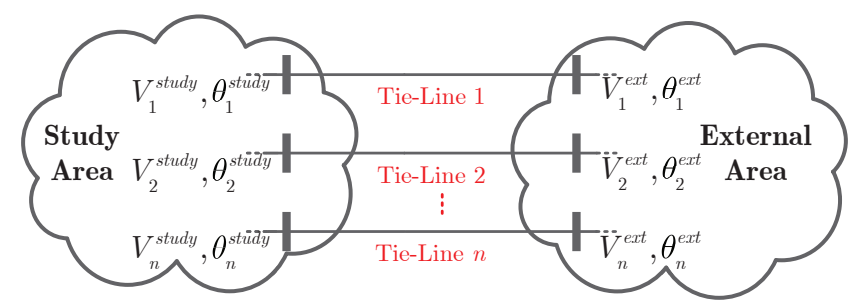

Fig. 1. The power system is divided into a study area and an external area, which is to be reduced.

The interface between the systems is defined by their $n$ tielines and buses. Given that the network with $N$ buses has an admittance matrix $Y=G+j B$, every bus satisfies the two power equations

$$
\begin{aligned}
P_{i}= & V_{i}^{2} G_{i i}+\sum_{j=1 ; j \neq i}^{N} V_{i} V_{j} B_{i j} \sin \left(\theta_{i}-\theta_{j}\right) \\
& +\sum_{j=1 ; j \neq i}^{N} V_{i} V_{j} G_{i j} \cos \left(\theta_{i}-\theta_{j}\right) \\
Q_{i}= & -V_{i}^{2} B_{i i}+\sum_{j=1 ; j \neq i}^{N} V_{i} V_{j} G_{i j} \sin \left(\theta_{i}-\theta_{j}\right) \\
& -\sum_{j=1 ; j \neq i}^{N} V_{i} V_{j} B_{i j} \cos \left(\theta_{i}-\theta_{j}\right)
\end{aligned}
$$

where $P_{i}$ and $Q_{i}$ are the injected real and reactive power respectively of bus $i$. This means that in order to have the equations (1) well-defined for the buses of the external area, it is required that it has the voltage magnitudes $V_{i}^{s t u d y}$ and phases $\theta_{i}^{\text {study }}$ that are adjacent to it as input signals. And having the external area output the voltage magnitudes $V_{i}^{\text {ext }}$ and phases $\theta_{i}^{e x t}$ will ensure that the study area has all the required bus variables available to make (1) well-defined for all its buses that are adjacent to the external area. The input and output signals are analogously defined for the study area.

Given this interface the external area can be linearized around a steady-state and reduced after which it is reconnected to the study area. The objective is to do the model reduction so that the dynamics of the nonlinear study area is affected as little as possible when replacing the nonlinear description of the external area with the reduced order linear model.

\section{Model Reduction Method}

Structured model order reduction is a model reduction technique that can be applied to systems composed of subsystems that are interconnected with some network dynamics. The idea is to reduce the model order of the subsystems while retaining the interconnection structure and keeping the global model error small. The general system (Fig. 2) to which structured model reduction can be applied is composed of $q$ subsystems described by the transfer function matrix

$$
G(s)=\left[\begin{array}{ccc}
G_{1}(s) & & 0 \\
& \ddots & \\
0 & & G_{q}(s)
\end{array}\right]=:\left[\begin{array}{c|c}
A_{G} & B_{G} \\
\hline C_{G} & D_{G}
\end{array}\right]
$$

with transfer functions $G_{k}(s) \in \mathbb{C}^{p_{k} \times m_{k}}$ and an interconnecting network

$$
N(s)=\left[\begin{array}{c|c}
E(s) & F(s) \\
\hline H(s) & K(s)
\end{array}\right]=:\left[\begin{array}{c|cc}
A_{N} & B_{N, 1} & B_{N, 2} \\
\hline C_{N, 1} & D_{E} & D_{F} \\
C_{N, 2} & D_{H} & D_{K}
\end{array}\right] .
$$

We want to find a reduced order system that approximates the mapping from $u_{1}^{N}$ to $y_{1}^{N}$ defined by the lower linear linear fractional transformation 


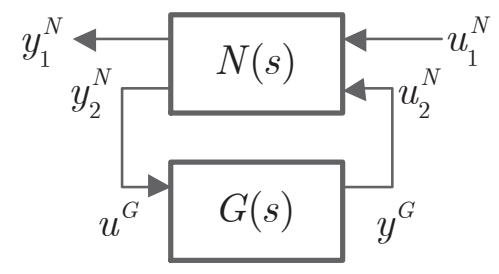

Fig. 2. The interconnected system. $G$ is the system that should be reduced and $N$ is the interconnecting network. $w$ and $z$ are external input and output respectively. $u$ and $y$ are the input and output to the system that should be reduced.

$$
\begin{aligned}
& \begin{array}{l}
\mathcal{F}_{L}(N, G):=E(s)+F(s)(I-G(s) K(s))-1 G(s) H(s)= \\
{\left[\begin{array}{cc|c}
A_{N}+B_{N, 2} L D_{G} C_{N, 2} & B_{N, 2} L C_{G} \\
B_{G} M C_{N, 2} & A_{G}+B_{G} M D_{K} C_{G} & B_{N, 1}+B_{N, 2} L D_{G} D_{H} \\
\hline C_{G} M D_{H} \\
\hline C_{N, 1}+D_{F} D_{G} M C_{N, 2} & D_{F} L C_{G} & D_{E}+D_{F} D_{G} M D_{H}
\end{array}\right]}
\end{array} \\
& =:\left[\begin{array}{c|c}
A & B \\
\hline C & D
\end{array}\right], \quad L:=\left(I-D_{G} D_{K}\right)^{-1}, \quad M:=\left(I-D_{K} D_{G}\right)^{-1} \text {, }
\end{aligned}
$$

with the constraint that the interconnecting network $N$ is retained, i.e. the objective is to find the reduced order system $\hat{G}$ such that

$$
\left\|\mathcal{F}_{l}(N, G)-\mathcal{F}_{l}(N, \hat{G})\right\|_{\infty}
$$

is made as small as possible and

$$
\hat{G} \in\left\{F(s): F(s)=\operatorname{diag}\left\{F_{1}(s), \ldots, F_{q}(s)\right\}\right\}
$$

where $F_{k}(s) \in \mathbb{C}^{p_{k} \times m_{k}}, k=1, \ldots, q$.

Finding the optimal minimum to (5) is very difficult, since it is a nonconvex optimization problem. We will therefore have to resort to suboptimal methods, which yield solutions satisfying the constraints while trying to minimize the norm of the model error. The model order reduction algorithm used in this paper is based on the idea of balanced truncation, see for example [22] and [27]. To enforce the structural constraints we use a generalization of balanced truncation as described in [28], [29], [25], [26]. The notation used here closely follows the one used in [25] and [26].

It uses the reachability and observability Gramians $P$ and $Q$ given by the Lyapunov equations

$$
A P+P A^{T}+B B^{T}=0, \quad A^{T} Q+Q A+C^{T} C=0,
$$

with the matrices $A, B, C$ defined by (4). It is helpful to use a partition with the blocks $Q_{N}, P_{N}$ for the interconnecting network that is not reduced and with the blocks $Q_{k}, P_{k}$ for subsystem $k$ that should be reduced separately, but in a way so that the closed-loop dynamics is retained.

$$
\begin{gathered}
Q=\left[\begin{array}{cc}
Q_{N} & Q_{N G} \\
Q_{N G}^{T} & Q_{G}
\end{array}\right], Q_{G}=\left[\begin{array}{ccc}
Q_{1} & \ldots & Q_{1 q} \\
\vdots & \ddots & \vdots \\
Q_{1 q}^{T} & \cdots & Q_{q}
\end{array}\right] \\
P=\left[\begin{array}{cc}
P_{N} & P_{N G} \\
P_{N G}^{T} & P_{G}
\end{array}\right], P_{G}=\left[\begin{array}{ccc}
P_{1} & \ldots & P_{1 q} \\
\vdots & \ddots & \vdots \\
P_{1 q}^{T} & \ldots & P_{q}
\end{array}\right]
\end{gathered}
$$

The method balances the subsystems $G_{k}(s)$ by the coordinate transformation $x_{k}=T_{k} \bar{x}_{k}$ that makes the transformed Gramians $\bar{Q}_{k}=T_{k}^{T} Q_{k} T_{k}$ and $\bar{P}_{k}=T_{k}^{-1} P_{k} T_{k}^{-T}$ subsystem balanced, which means that

$$
\begin{aligned}
& \bar{P}_{k}=\bar{Q}_{k}=\Sigma_{k}=\operatorname{diag}\left\{\sigma_{k, 1}, \ldots, \sigma_{k, n_{k}}\right\}, \\
& \sigma_{k, 1} \geq \ldots \geq \sigma_{k, n_{k}}, \\
& \sigma_{k, j}=\sqrt{\lambda_{j}\left(P_{k} Q_{k}\right)}=\sqrt{\lambda_{j}\left(\bar{P}_{k} \bar{Q}_{k}\right)} .
\end{aligned}
$$

Thus if the original state vector has the structure,

$$
x=\left[\begin{array}{llll}
x_{N}^{T} x_{1}^{T} & \ldots & x_{q}^{T}
\end{array}\right]^{T},
$$

then the transformed system will have the states $\bar{x}$ defined by

$$
T \bar{x}=x,
$$

where

$$
T=\operatorname{diag}\left(T_{N}, T_{1}, \ldots, T_{q}\right), T_{N} \in \mathbb{R}^{n_{N} \times n_{N}}, T_{k} \in \mathbb{R}^{n_{k} \times n_{k}},
$$

and $n_{N}$ and $n_{k}$ are the order of system $N$ and $G_{k}$ respectively.

Having made a coordinate transformation, either truncation or singular perturbation is used calculate the reduced order systems $\hat{G}_{i}$ of the subsystems in $G$. To this end the structured Hankel singular values can guide the choice of which states to retain as explained below.

The strength of the structured model reduction algorithm is that the block-diagonal elements of the Gramians defined by (6) tell us how reachable and observable the states of the subsystems are when we control the global input signal $u_{1}^{N}$ and observe the global output signal $y_{2}^{N}$ (Fig. 2). More precisely, the following holds [25]:

If all states excepts the ones in subsystem $k$ are zero at time zero, then

$$
\left\|y_{1}^{N}(t)\right\|_{[0, \infty]}^{2}=x_{k}(0)^{T} Q_{k} x_{k}(0) .
$$

and assuming that all states of the interconnected system are zero at $t=-\infty$ and that we would like to control the states of subsystem $k$ to the specific state $x_{k}(0)=x_{k}^{*}$, while the states $x_{N}(0)$ and $x_{i}(0), i \neq k$ are free variables, then the minimum control signal satisfies

$$
\begin{gathered}
\min _{\substack{u_{1}^{N} \in L_{2}(-\infty, 0) \\
x(0) \in X_{k}^{*}}}\left\|u_{1}^{N}(t)\right\|_{[-\infty, 0]}^{2}=\left(x_{k}^{*}\right)^{T} P_{k}^{-1} x_{k}^{*}, \\
X_{k}^{*}=\left\{x: x \text { has structure }(10) \text { and } x_{k}=x_{k}^{*}\right\} .
\end{gathered}
$$

\section{Structured Model Reduction of Power SYSTEMS}

The structured model reduction algorithm accounted for in Section III is based on the notion of dividing the system into the subsystems $N$ and $G$. This makes it suitable for application to power systems with one study area, which we want to retain a non-linear description of and one external area which we want to reduce. We will henceforth assume that the system $G$ only consists of one subsystem that we 
want to reduce, i.e. with the notation introduced in Section III, $q=1$. Although we make this assumption, there is nothing preventing $G$ from being composed of more than one subsystem. We now propose the following four-step algorithm:

\section{Defining the model}

A general power system will be described by differential algebraic equations (DAE) of the form

$$
\begin{aligned}
& \dot{x}=f\left(x, x_{\mathrm{alg}}, u\right) \\
& 0=g\left(x, x_{\mathrm{alg}}, u\right) .
\end{aligned}
$$

The states $x$ will be describing the generators, controllers, etc., whereas the algebraic variables $x_{\text {alg }}$ will be the voltages and phases of the buses as well as algebraic variables describing the generators, controllers, etc. The signal $u$ will be an exogenous input to the power system. It could for instance describe time-varying loads. Divide this system into a study area, denoted $N$ and an external area denoted $G$. A general system with this structure can be described by the DAEs

$$
\begin{aligned}
& \dot{x}^{G}=f^{G}\left(x^{G}, x_{\text {alg }}^{G}, u^{G}\right) \\
& 0=g^{G}\left(x^{G}, x_{\text {alg }}^{G}, u^{G}\right)
\end{aligned}
$$

and

$$
\begin{aligned}
& \dot{x}^{N}=f^{N}\left(x^{N}, x_{\mathrm{alg}}^{N}, u_{1}^{N}, u_{2}^{N}\right) \\
& 0=g^{N}\left(x^{N}, x_{\mathrm{alg}}^{N}, u_{1}^{N}, u_{2}^{N}\right)
\end{aligned}
$$

The variables $u^{G}$ and $u_{2}^{N}$ are the voltage magnitudes and phases of the buses at the tie-line as described in Section II and $u_{1}^{N}$ is the same exogenous input as in (14), (Fig. 2).

\section{Linearizing}

In order to apply the structured model reduction algorithm described in Section III it is first necessary to linearize both the study area and the external area. By solving the power flow problem, the steady-state of the power system is acquired around which the linearization is done. The linearization of $(15,16)$ will take the form

$$
\begin{gathered}
\left(\begin{array}{l}
\dot{x}^{G} \\
0
\end{array}\right)=\left(\begin{array}{cc}
A_{11}^{G} & A_{12}^{G} \\
A_{21}^{G} & A_{22}^{G}
\end{array}\right)\left(\begin{array}{c}
x^{G} \\
x_{\mathrm{alg}}^{G}
\end{array}\right)+\left(\begin{array}{c}
B_{1}^{G} \\
B_{2}^{G}
\end{array}\right) u^{G} \\
\left(\begin{array}{l}
\dot{x}^{N} \\
0
\end{array}\right)=\left(\begin{array}{cc}
A_{11}^{N} & A_{12}^{N} \\
A_{21}^{N} & A_{22}^{N}
\end{array}\right)\left(\begin{array}{c}
x^{N} \\
x_{\mathrm{alg}}^{N}
\end{array}\right)+\left(\begin{array}{ll}
B_{11}^{N} & B_{12}^{N} \\
B_{21}^{N} & B_{22}^{N}
\end{array}\right)\left(\begin{array}{c}
u_{1}^{N} \\
u_{2}^{N}
\end{array}\right) .
\end{gathered}
$$

\section{Nonlinear model}

With the system $G$ being reduced to

$$
\hat{G}(s)=\left[\begin{array}{c|c}
A_{\hat{G}} & B_{\hat{G}} \\
\hline C_{\hat{G}} & D_{\hat{G}}
\end{array}\right]
$$

it can be reconnect to the non-linear description of the study area yielding the reduced interconnected system

$$
\begin{aligned}
& \dot{x}^{\hat{G}}=A_{\hat{G}} x^{\hat{G}}+B_{\hat{G}} u^{\hat{G}} \\
& u_{2}^{N}=y_{\hat{G}}=C_{\hat{G}} x^{\hat{G}}+D_{\hat{G}} u^{\hat{G}} \\
& \dot{x}^{N}=f^{N}\left(x^{N}, x_{\mathrm{alg}}^{N}, u_{1}^{N}, u_{2}^{N}\right) \\
& 0=g^{N}\left(x^{N}, x_{\mathrm{alg}}^{N}, u_{1}^{N}, u_{2}^{N}\right) \\
& u^{\hat{G}}=y_{2}^{N}=M_{N} x_{\mathrm{alg}}^{N} .
\end{aligned}
$$

$$
\begin{aligned}
& \dot{x}^{N}=\left(A_{11}^{N}-A_{12}^{N} A_{22}^{N^{-1}} A_{21}^{N}\right) x^{N} \\
& +\left[\begin{array}{ll}
\left(B_{11}^{N}-A_{12}^{N} A_{22}^{N^{-1}} B_{21}\right) & \left(B_{12}^{N}-A_{12}^{N} A_{22}^{N^{-1}} B_{22}\right)
\end{array}\right]\left(\begin{array}{c}
u_{1}^{N} \\
u_{2}^{N}
\end{array}\right) \\
& \left(\begin{array}{l}
y_{1}^{N} \\
y_{2}^{N}
\end{array}\right)=\left(\begin{array}{l}
0 \\
M^{N}
\end{array}\right)\left(-A_{22}^{N^{-1}}\left(A_{21}^{N} x^{N}+B_{21}^{N} u_{1}^{N}+B_{22}^{N} u_{2}^{N}\right)\right) \\
& +\left(\begin{array}{ll}
0 & I \\
0 & 0
\end{array}\right)\left(\begin{array}{c}
u_{1}^{N} \\
u_{2}^{N}
\end{array}\right)
\end{aligned}
$$

We can note that the system $N$ has one input signal $u_{1}^{N}$

\section{. Model reduction}

equations for the interconnected system is readily found $P_{G}$ and $Q_{G}$ from the matrices (8) and (7) a change of structured Hankel singular values (9) the model order can be

What makes these systems easy to work with is that the algebraic variables $x_{G}^{a l g}$ and $x_{N}^{a l g}$ can be solved for

$$
\begin{gathered}
x_{\mathrm{alg}}^{G}=-A_{22}^{G^{-1}}\left(A_{21}^{G} x^{G}+B_{2}^{G} u^{G}\right) \\
x_{\mathrm{alg}}^{N}=-A_{22}^{N^{-1}}\left(A_{21}^{N} x^{N}+B_{21}^{N} u_{1}^{N}+B_{22}^{N} u_{2}^{N}\right) .
\end{gathered}
$$

If the matrices $M^{G}$ and $M^{N}$ select which algebraic variables the two subsystems output, i.e. the tie-line voltage magnitudes and phases, the DAEs can be recast into the following ordinary differential equations

\section{A. Algorithm preferences}

Having linearized the system, we want to find the reduced system $\hat{G}$ that makes (5) as small as possible. The outcome of the model reduction will of course be dependent on the choice of exogenous input signals $u_{1}^{N}$ and output signals $y_{1}^{N}$. In the example that will be used to demonstrate the algorithm the input signals $u_{1}^{N}$ were chosen as the loads of the buses 5 and 6 (Fig. 3). This choice could be of interest if one wants to design a power system stabilizer (PSS) at the generator connected 
to bus 1 that attenuates oscillations following load variations. However, the choice should be application dependent.

The most natural choice for the output variables $y_{1}^{N}$ is to choose them as the voltage magnitudes and phases of the buses of the external area at the tie-lines. This makes a good choice considering how we interconnect the two areas. Assuming that the reduced order external system exhibited the same voltage amplitudes and phases on the buses at the tie-lines as the full external system, then it would follow that the trajectories of the study system of both models would be identical, given that they had the same initial condition. Therefore if we can make the tie-line variables of the reduced system and the full system be close to each other, then the two study systems should have similar trajectories which is the objective of the model reduction.

\section{B. Required assumptions}

The structured model reduction algorithm, which is based on balanced truncation, require that the interconnected system is asymptotically stable, since the reachability and observability Gramians will not be well defined otherwise. A power system with only synchronous generators and loads will however always have an eigenvalue at the origin as long as no reference for the voltage phase angles is chosen. There are a number of different approaches that can be taken to accommodate for this. One way is to simply fix one of the generators to be the reference in a similar manner to the selection of a slack bus in the power flow problem [30].

Another possibility that may arise is that the power system in question contains an infinite bus that models an inert system which dynamics are unknown or simply disregarded. This could for instance be a neighboring country connected by a tieline. If this is the case, the system will not have an eigenvalue at the origin and the Gramians are well defined.

A third option would be to choose a reference angle to remove the zero eigenvalue, do the model reduction and then revert the system to one without a reference. This procedure might however yield unstable dynamics since the reduced system will have an eigenvalue in the proximity of the origin, and if it is not being paid attention to, it might end up in the right half plane.

\section{Algorithm DEMONSTRATION}

To calculate the reduced order power system we have used the Power System Analysis Toolbox (PSAT) [31]. Its Simulink interface can be used to draw up the two subsystems $N$ and $G$. After using the built-in method to solve the power flow problem, PSAT can return the two linearized subsystems.

Then to simulate the full system and the reduced order system at the presence of some perturbation, MATLAB's ode15s solver for stiff DAEs of index 1 was used. This particular solver was chosen because power systems with highorder generators and Automatic Voltage Regulators (AVR) are known to have both really fast and slow dynamics, i.e. they are stiff systems [32].

\section{A. Model}

The system that will be used to demonstrate the structured model reduction algorithm is the WSCC 3-machine, 9-bus system (Fig. 3) [33] with a connected infinite bus. We will choose the study area $N$ to be defined by the generator connected to bus 1 and the buses 1, 4, 5 and 6 , whereas the external area $G$ is selected to be composed of the generators connected to buses 2 and 3 respectively as well as the remaining buses. The synchronous machines are modeled as fourth order systems with connected AVRs of the standard IEEE model 1. The DAEs for each machine are

$$
\begin{aligned}
\dot{\delta} & =\Omega_{b}(\omega-1) \\
\dot{\omega} & =\left(p_{m}-p_{e}-D(\omega-1)\right) / M \\
\dot{e_{q}^{\prime}} & =\left(-e_{q}^{\prime}-\left(x_{d}-x_{d}^{\prime}\right) i_{d}+v_{f}\right) / T_{d 0}^{\prime} \\
\dot{e_{d}^{\prime}} & =\left(-e_{d}^{\prime}+\left(x_{q}-x_{q}^{\prime}\right) i_{q}\right) / T_{q 0}^{\prime} \\
0 & =v_{q}+r_{a} i_{q}-e_{q}^{\prime}+x_{d}^{\prime} i_{d} \\
0 & =v_{d}+r_{a} i_{d}-e_{d}^{\prime}-x_{q}^{\prime} i_{q} \\
0 & =-v_{d}+v \sin (\delta-\theta) \\
0 & =-v_{q}+v \cos (\delta-\theta) \\
0 & =-p_{e}+\left(v_{q}+r_{a} i_{q}\right) i_{q}+\left(v_{d}+r_{a} i_{d}\right) i_{d}
\end{aligned}
$$

where we recognize $\delta$ to be the synchronous machine internal angle and $\omega$ to be the angular velocity of the machine [30]. These equations hold assuming that the field saturation characteristic is linear and that the field voltage $v_{f}$ has no feedback term depending on the rotor speed and active power produced by the machine. The AVRs are described by the DAEs

$$
\begin{aligned}
& \dot{v}_{m}=\left(v-v_{m}\right) / T_{r} \\
& \dot{v}_{r 1}=\left(K_{a}\left(v_{r e f}-v_{m}-v_{r 2}-\frac{K_{f}}{T_{f}} v_{f}\right)-v_{r 1}\right) / T_{a} \\
& v_{r}= \begin{cases}v_{r 1} & \text { if } v_{r}^{\text {min }} \leq v_{r 1} \leq v_{r}^{\text {max }} \\
v_{r}^{\text {max }} & \text { if } v_{r 1}>v_{r}^{\text {max }} \\
v_{r}^{\text {min }} & \text { if } v_{r 1}<v_{r}^{\text {min }}\end{cases} \\
& \dot{v}_{r 2}=-\left(\frac{K_{f}}{T_{f}} v_{f}+v_{r 2}\right) / T_{f} \\
& \dot{v}_{f}=-\left(v_{f}\left(K_{e}+S_{e}\left(v_{f}\right)\right)-v_{r}\right) / T_{e}
\end{aligned}
$$

with

$$
S_{e}\left(v_{f}\right)=A_{e} e^{B_{e}\left|v_{f}\right|}
$$

The different machines of the power system are connected through transmission lines. Each bus satisfies the algebraic equations (1) and thereby interconnect the generators.

\section{B. Results}

To evaluate the structured model order reduction algorithm it will be compared with ordinary balanced truncation applied to the external system $G$. We will introduce a perturbation in the internal generator angle $\delta$ of generator 1 to determine the reduced order model's ability to capture the transients 


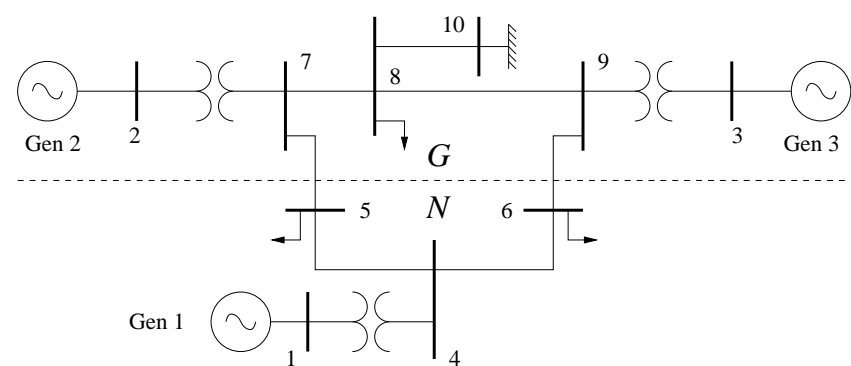

Fig. 3. The WSCC 3-machine, 9-bus system with an infinite bus.

induced by the perturbation. This is important if one wishes to study how well damped the system is. A reduced order model with this quality could for instance be used to design a power system stabilizer (PSS) controller that dampens interarea oscillations.

To decide what the model order of the reduced system should be, we can use the structured Hankel singular values (9). Although nothing conclusive can be inferred from them, they can be used for guidance. Fig. 4 shows that the size of the structured Hankel singular values is dropping quickly, indicating that there is a good chance the system can be modeled with only a few states. It turns out that at least three states are required to model the external area with satisfactory results.



Fig. 4. The structured Hankel singular values of system $G$ in a semilog scale.

The output variables $y_{1}^{N}$ of the interconnected system that we try to model accurately are the voltage amplitudes and phases of the tie-lines buses. Fig. 5 and 6 show the transients of $V_{7}$ and $\theta_{7}$ when the internal generator angle $\delta$ of generator 1 is perturbed $1.6 \%$ and the external area has been reduced from 16 states to 3 . We see that the reduced model based on structured model reduction captures most of the interesting dynamics although it misses some in the higher frequencies. If ordinary balanced truncation is applied to reduce the external area to a system of order 3, then when interconnecting the reduced system $\hat{G}$ with the study area, we see that the system becomes unstable. Similar observations can be made for bus 9 .

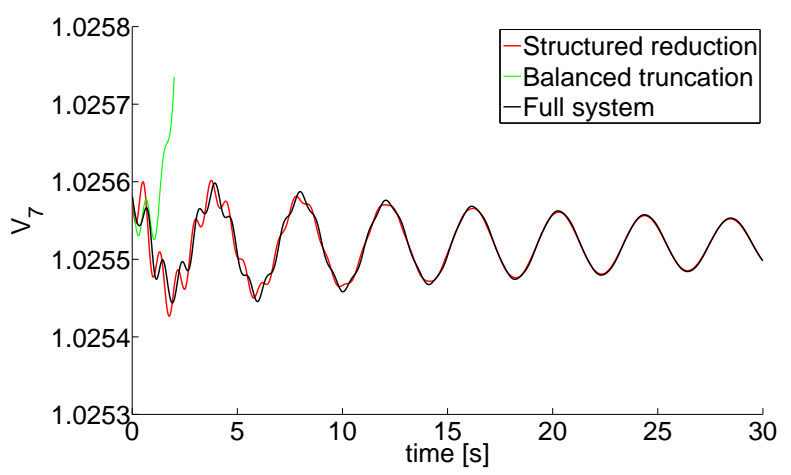

Fig. 5. Transients of $V_{7}$ at the tie-line bus with a third order system $\hat{G}$. Structured model reduction and ordinary balanced truncation is compared with the full system.

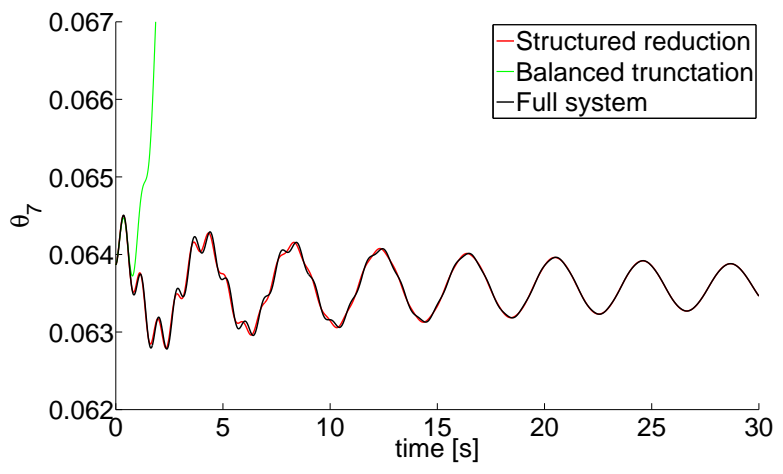

Fig. 6. Transients of $\theta_{7}$ at the tie-line bus with a third order system $\hat{G}$. Structured model reduction and ordinary balanced truncation is compared with the full system.

To get an even better model approximation we can just add a few more states. Looking at Fig. 4 we see that there is a drop between the seventh and eighth structured Hankel singular value. This suggests adding four more states to the reduced model (Fig. 7, 8) to capture the high frequency dynamics that was lost in the reduced system with $\hat{G}$ of order 3 . Doing this we see that the reduced model derived from the application of the structured model order reduction algorithm almost achieves a perfect fit with the full model whereas the application of ordinary balanced truncation yields a sizeable error.

\section{CONCLUSION}

We have proposed an algorithm for applying structured model order reduction to power systems divided into an external area that should be reduced and a study area. The resulting reduced order system is an interconnection of a reduced order linear system describing the external area and the nonlinear study area. The algorithm was demonstrated on the WSCC 3-machine, 9-bus system. It was found that only three states were required to describe the 16 states external system, when using the proposed algorithm. Ordinary balanced 


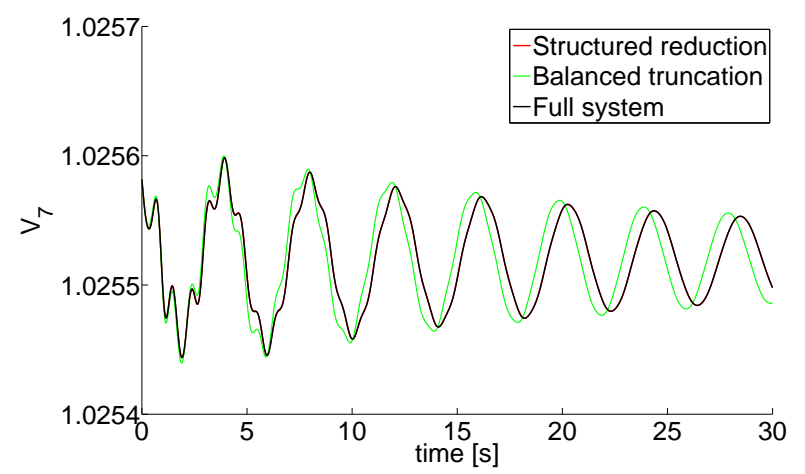

Fig. 7. Transients of $V_{7}$ at the tie-line bus with a seventh order system $\hat{G}$.

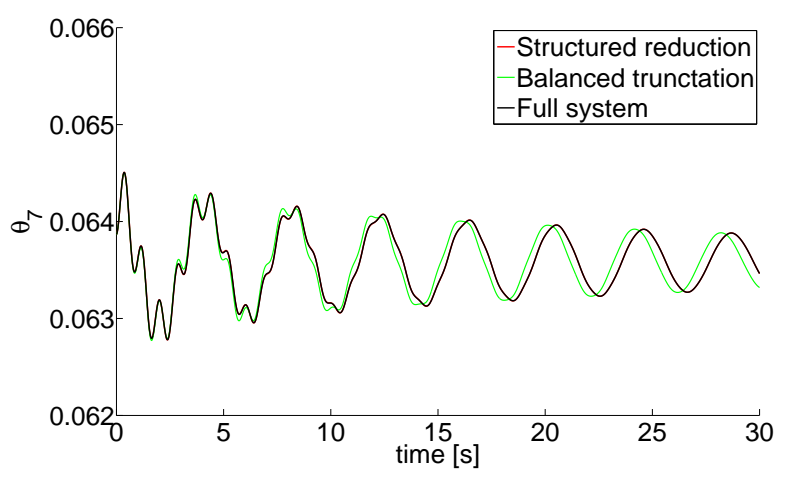

Fig. 8. Transients of $\theta_{7}$ at the tie-line bus with a seventh order system $\hat{G}$.

truncation was also applied to the external area to illustrate the importance of taking the full system into account when doing a local model reduction. In this case the reduction of the external area to a system of order three yielded an unstable system.

\section{REFERENCES}

[1] J. H. Chow, G. Peponides, P. V. Kokotovic, B. Avramovic, and J. R. Winkelman, Time-Scale Modeling of Dynamic Networks with Applications to Power System, J. H. Chow, Ed. New York: Springer-Verlag, 1982.

[2] M. Oatts, S. Erwin, and J. Hart, "Application of the REI equivalent for operations planning analysis of interchange schedules," in Power Industry Computer Application Conference, 1989. PICA '89, Conference Papers, may 1989, pp. $424-430$.

[3] G. Rogers, Power System Oscillations. Norwell, MA: Kluwer Academic Publishers, 1999.

[4] R. Podmore, "Development of dynamic equivalents for transient stability studies," EPRI, Report EPRI Project 763, May 1997.

[5] F. Milano and S. K., "Dynamic REI equivalents for short circuit and transient stability analysis," Electric Power Systems Research, vol. 79, pp. 878-887, 2009.

[6] A. Bose, "Modeling of External Networks for On-Line Security Analysis," IEEE Transactions on Power Apparatus and Systems, vol. PAS-103, pp. 2117-2125, Aug. 1984.

[7] J. Sanchez-Gasca, J. Chow, and R. Galarza, "Reduction of linearized power systems for the study of interarea oscillations," in Proceedings of the 4th IEEE Conference on Control Applications, 1995., sep 1995, pp. $624-630$.

[8] J. Undrill, J. Casazza, E. Gulachenski, and L. Kirchmayer, "Electromechanical Equivalents for use in Power System Stability Studies," IEEE Transactions on Power Apparatus and Systems, vol. PAS 90, pp. 2060 2071, 1971.
[9] R. W. de Mello, R. Podmore, and S. K. N., "Coherency-Based Dynamic Equivalents: Applications in Transient Stability Studies," Proceedings of PICA Conference, pp. 23-31, 1975.

[10] A. Morched and V. Brandwajn, "Transmission network equivalents for electromagnetic transients studies," IEEE Transactions on Power Apparatus and Systems, vol. PAS-102, no. 9, pp. 2984 -2994, sept. 1983.

[11] J. Sanchez-Gasca and J. Chow, "Power system reduction to simplify the design of damping controllers for interarea oscillations," IEEE Transactions on Power Systems, vol. 11, no. 3, pp. $1342-1349$, aug 1996.

[12] A. J. Germond and R. Podmore, "Dynamic Aggregation of Generating Unit Models," IEEE Transactions on Power Apparatus and Systems, no. 4, pp. 1060-1069, 1978.

[13] J. Chow, R. Galarza, P. Accari, and W. Price, "Inertial and slow coherency aggregation algorithms for power system dynamic model reduction," IEEE Transactions on Power Systems, vol. 10, no. 2, pp. $680-685$, may 1995.

[14] R. A. Date and J. H. Chow, "Aggregation Properties of Linearized Two-Time-Scale Power Networks," IEEE Transactions on Circuits and Systems, vol. 38, no. 7, pp. 720-730, Jul. 1991.

[15] J. H. Chow, "A Nonlinear Model Reduction Formulation for Power System Slow Coherency and Aggregation," in Proceedings of Workshop on Advances in Control and its Applications, ser. Lecture Notes in Control \& Information Sciences, P. I. H.K. Khalil, J.H. Chow, Ed., vol. 208, 1996.

[16] —, "New Algorithms for Slow Coherency Aggregation of Large Power Systems," in Systems and Control Theory for Power Systems, ser. IMA Volumes in Mathematics and its Applications, R. J. T. Joe H. Chow, Petar V. Kokotovic, Ed., vol. 64, Institute for Mathematics and its Applications (IMA). Springer-Verlag, 1995, pp. 95-115.

[17] R. Galarza, J. Chow, W. Price, A. Hargrave, and P. Hirsch, "Aggregation of exciter models for constructing power system dynamic equivalents," IEEE Transactions on Power Systems, vol. 13, no. 3, pp. 782 -788, aug 1998.

[18] M. Brucoli, M. La Scala, N. Pitrone, and M. Trovato, "Dynamic modelling for retaining selected portions of interconnected power networks," IEE Proceedings on Generation, Transmission and Distribution, vol. 135, no. 2, pp. $118-123$, mar 1988.

[19] M. Ibrahim, O. Mostafa, and A. El-Abiad, "Dynamic equivalents using operating data and stochastic modeling," IEEE Transactions on Power Apparatus and Systems, vol. 95, no. 5, pp. 1713 - 1722, sept. 1976.

[20] D. Chaniotis and M. Pai, "Model reduction in power systems using krylov subspace methods," Power Systems, IEEE Transactions on, vol. 20, no. 2, pp. 888-894, 2005.

[21] P. Parrilo, S. Lall, F. Paganini, G. Verghese, B. Lesieutre, and J. Marsden, "Model reduction for analysis of cascading failures in power systems," in American Control Conference, 1999. Proceedings of the 1999, vol. 6. IEEE, 1999, pp. 4208-4212.

[22] D. Enns, "Model reduction with balanced realizations: an error bound and a frequency weighted generalization," in Proceedings of the IEEE Conference on Decision and Control, Las Vegas, Nevada, 1984.

[23] B. Anderson and Y. Liu, "Controller reduction: Concepts and approaches," IEEE Transactions on Automatic Control, vol. 34, pp. 802812, 1989.

[24] L. Li and F. Paganini, "Structured coprime factor model reduction based on LMIs," Automatica, vol. 41, no. 1, pp. 145-151, Jan. 2005.

[25] A. Vandendorpe and P. Van Dooren, Model Order Reduction: Theory, Research Aspects and Applications, ser. Mathematics in Industry Series. Springer Verlag, 2007, ch. Model reduction of interconnected systems.

[26] H. Sandberg and R. M. Murray, "Model reduction of interconnected linear systems," Optimal Control, Applications and Methods, Special Issue on Directions, Applications, and Methods in Robust Control, vol. $30: 3$, pp. 225-245, 2009.

[27] K. Glover, "All optimal Hankel-norm approximations of linear multivariable systems and their $L_{\infty}$-error bounds," International Journal of Control, vol. 39, pp. 1115-1193, 1984.

[28] G. Schelfhout and B. De Moor, "A note on closed-loop balanced truncation," IEEE Transactions on Automatic Control, vol. 41, pp. 14981500, 1996.

[29] P. Wortelboer, "Frequency-weighted balanced reduction of closed-loop mechanical servo-systems: theory and tools," Ph.D. dissertation, TU Delft, Delft University of Technology, 1994-06-20. 
[30] P. Kundur, Power system stability and control, N. Balu and M. Lauby, Eds. McGraw-Hill New York, 1994.

[31] F. Milano, "An Open Source Power System Analysis Toolbox," vol. 20, no. 3, pp. 1199-1206, Aug. 2005.

[32] J. Machowski, J. Bialek, and J. Bumby, Power system dynamics: stability and control. John Wiley \& Sons Inc, 2008.

[33] P. Anderson, A. Fouad, I. of Electrical, and E. Engineers, Power system control and stability. Iowa State University Press, 1986. 